\title{
AN ELEMENTARY PROPERTY OF BOUNDED DOMAINS*
}

\author{
BY W. L. AYRES $\dagger$
}

It is the purpose of this note to prove the following property of a bounded domain $\ddagger$ lying in a two-dimensional euclidean space.

Theorem. If $P$ is a point of a plane and bounded domain, there exists a triangle such that it contains $P$ in its interior, has its vertices on the boundary of the domain, and every other point of the triangle and its interior lie in the domain.

Proof. Let $D$ be the domain and $B$ denote the boundary of $D$. Let $r$ denote the lower limit of all numbers $d(P, x)$, where $x$ is any point of the boundary $B$ and $d(P, x)$ denotes the distance from $P$ to $x$. Let $C$ be the circle with center at $P$ and radius $r$. Since $B$ is a closed set, the circle $C$ contains a point $X$ of $B$. Let $E_{1}$ and $E_{2}$ be points whose distance from $P$ is $\frac{1}{2} r$ and which lie on the line through $P$ perpendicular to the line $X P$. Let $E_{i} F_{i}(i=1,2)$ be a ray through $E_{i}$ parallel to the ray $X P$.§ If $y$ is any point of the ray $X P$ such that $d(P, y) \geqq r$, let $C_{y}$ be the circle with center at $P$ and

* Presented to the Society, October 29, 1927.

$\dagger$ National Research Fellow in Mathematics.

$¥$ A connected set of points $D$ is said to be a domain if for every point $P$ of $D$ there is a circle with center at $P$, such that $D$ contains every point in the interior of the circle. The boundary of a domain is the set of all limit points of the domain which do not belong to the domain. The boundary of a bounded domain is a closed and bounded point set, and if $H$ is any connected point set containing a point of the domain and a point not belonging to the domain, then $H$ contains a point of the boundary of the domain.

$\S$ If $l$ is a line and $A$ and $B$ are points of $l$, the ray $A B$ (but not the ray $B A$ ) is the maximal connected subset of $l-A$ which contains $B$. If $A B$ is a ray and $C$ is a point not on the line $A B$ then if $l$ denotes the line through $C$ parallel to the line $A B$ the ray through $C$ parallel to the ray $A B$ is the maximal connected subset of $l-C$ which lies on the $B$-side of the line $A C$. 
radius $d(P, y)$ and let $y_{i}$ be the intersection of $C_{y}$ and the ray $E_{i} F_{i}$. Let $[y]$ be the set of all points $y$ of the ray $X P$ such that $d(P, y) \geqq r$ and the arc $y_{1} y y_{2}$ of $C_{y}$ contains a point of the boundary $B$. Using the fact that the boundary $B$ is closed and the ray $X P$ contains at least one point of $B$, it is not difficult to show that the set $[y]$ is non-vacuous and closed. Since $[y]$ is closed, there is a first point $A$ of $[y]$ on the ray $X P{ }^{*}$ The arc $A_{1} A A_{2}$ of $C_{A}$ contains a point $Y$ of the boundary $B$ and every point of the segment $X Y$ belongs to the domain $D$. There are two cases to consider according as $Y=A$ or $Y \neq A$.

Case I. If $Y \neq A$, let $Q$ be the intersection of the line $X Y$ and the line through $P$ parallel to the line $A Y$. Let $[z]$ be the set of all points $z$ of the ray $Q P$ such that either (a) the segment $X z$ contains a point of the boundary $B$, or (b) the segment $Y z$ contains a point of $B$, or (c) the point $z$ belongs to $B$. It follows easily that the set $[z]$ is non-vacuous, closed and contains no point of the interval $Q P$. Let $G$ be the first point of the set $[z]$ on the ray $Q P$. Then either $G$ is a point of the boundary $B$ or the segment $X G$ or $Y G$ contains a point of the boundary $B$.

Since $G$ does not lie on the interval $Q P$, the point $P$ is in the interior of the triangle $X Y G$, and every point in the interior of this triangle lies in the domain $D$. If the segment $X G$ contains a point of $B$, let $Z$ be the first point of $B$ on the ray $X G$. We may show that there is a first point of $B$ on the ray $X G$ as follows. Since the ray $X G$ makes an acute angle with the ray $X P$, the ray $X G$ contains a point $H$ of the circle $C$. Now the points of $B$ on the ray $X G$ form a set which is closed except possibly for the point $X$. But $X$ is not a limit point of the set of points of $B$ which lie on the ray $X G$, since no point of the segment $X H$ belongs to $B$. Hence the points of $B$ which lie on the ray $X G$ form a closed set and there is a first point $Z$ of $B$ on the segment $X G$ in the order $X$ to $G$.

* If $K$ is a set of points lying on a ray $A B$, a point $p$ of $K$ is said to be the first point of $K$ on the ray $A B$ if for every point $q$ of $K$ distinct from $p$ the point $p$ lies on the segment $A q$. 
Let $l$ be the line through $X$ parallel to the line $A Y$ and let $K$ denote the point of intersection of $l$ and the circle $C$ which is distinct from $X$ (the line $l$ is not tangent to the circle $C$ since $A \neq Y$ ). The segment $Y K$ contains the point $P$ and there is no point of $B$ on or inside the triangle $X Y K$ except possibly its vertices. Hence the angle $X Y Z$ is greater than the angle $X Y K$ and so $P$ lies in the angle $X Y Z$. Similarly the angle $Y X Z$ is greater than the angle $Y X A$ and so $P$ lies in the angle $Y X Z$. Then $P$ lies in the interior of the triangle $X Y Z$. The segment $X Z$ belongs to the domain $D$, since the segment $X H$ belongs to the domain $D$ and $Z$ is the first point of $B$ on the ray $X G$. The segment $Y Z$ and the interior of the triangle $X Y Z$ belong to the domain $D$, since they are subsets of the interior of the triangle $X Y G$. Therefore the triangle $X Y Z$ satisfies all the conditions of our theorem and is the desired triangle.

If no point of the segment $X G$ belongs to the boundary $B$, but the segment $Y G$ contains at least one point of $B$, then if $Z$ is chosen as the first point of $B$ on the ray $Y G$, the same proof shows that the triangle $X Y Z$ is the desired triangle. If neither the segment $X G$ nor the segment $Y G$ contains a point of $B$, the triangle $X Y G$ satisfies all the conditions of our theorem and is the required triangle.

Case II. If $A=Y$, let $l$ be the line through $P$ perpendicular to the line $X Y$ and let $G_{1}$ and $G_{2}$ be points of $l$ such that $P$ is between $G_{1}$ and $G_{2}$. On the ray $P G_{1}$, let $[z]$ denote the set of all points $z$ such that $z$ belongs to the boundary $B$ or the segment $X z$ contains a point of $B$ or the segment $Y z$ contains a point of $B$. It is easily seen that $[z]$ is non-vacuous and closed. Let $H$ be the first point of the set $[z]$ on the ray $P G_{1}$. The set of points of $B$ which lie on the ray $X H$ is closed except possibly for the point $X$. Since the angle $Y X H$ is acute, there is a segment $X K$ which lies entirely in the interior of the circle $C$ and thus entirely in the domain $D$. Then the set of points of $B$ on the ray $X H$ is closed. If the segment $X H$ contains a point of $B$, let $Z$ be the first point of $B$ on the segment $X H$ in the order $X$ to $H$. If the segment $X H$ con- 
tains no point of $B$ but the segment $Y H$ contains a point of $B$, let $Z$ be the first point of $B$ on the segment $Y H$ in the order $Y$ to $H$. There is a first point since the set of points of $B$ which lie on the ray $Y H$ is closed. If neither the segment $X H$ nor the segment $Y H$ contains a point of the boundary $B$, let $H$ be the point $Z$. In any case the triangle $X Y Z$ has its vertices on the boundary $B$ and every other point of the triangle and its interior belongs to the domain $D$, the segment $X Y$ contains the point $P$, and the angles $X Y Z$ and $Y X Z$ are acute.

In exactly the same manner, we determine a point $W$ of the boundary $B$ on the $G_{2}$-side of the line $X Y$, such that the triangle $X Y W$ has the same properties as the triangle $X Y Z$, where $Z$ is replaced by $W$.

Since the angles $X Y Z, Y X Z, X Y W$ and $Y X W$ are all acute, every point of the segment $Z W$ lies in the interior of the quadrilateral $X Z Y W$. If the segment $Z W$ does not contain the point $P$, the point $P$ lies in the interior of the triangle $X Z W$ or $Y Z W$. In this case the triangle $X W Z$ or the triangle $Y W Z$ (whichever contains $P$ in its interior) is the desired triangle. If the segment $W Z$ contains the point $P$, we have a quadrilateral $X Z Y W$ having its vertices on the boundary $B$ and every other point of the quadrilateral and its interior belonging to the domain $D$, and such that $P$ is the intersection of the diagonals of $X Z Y W$. Either the rays $X Z$ and $W Y$ have no point in common or the rays $Z X$ and $Y W$ have no point in common. Let us suppose that the rays $X Z$ and $W Y$ have no point in common. If the lines $X Z$ and $W Y$ are parallel, let $P Q$ be the ray through $P$ parallel to the ray $X Z$. If the lines $X Z$ and $W Y$ intersect in a point $M$, let $Q$ be a point of the line $P M$ such that $P$ is between $Q$ and $M$. On the ray $P Q$, let $[v]$ be the set of all points $v$ such that $v$ is a point of $B$ or the segment $X v$ contains a point of $B$ or the segment $W v$ contains a point of $B$. As above, we may show that the set $[v]$ is non-vacuous and closed. Let $N$ be the first point of the set $[v]$ on the ray $P Q$. If the segment $X N$ contains a point of $B$, let $V$ be the first point of the boun- 
dary $B$ on the ray $X N$. There is a first point $V$ since the set of points of $B$ which lie on the ray $X N$ is a closed set. Since $V$ is outside the quadrilateral $X Z Y W$, the angle $X W V$ is greater than the angle $X W Z$ and the angle $W X V$ is greater than the angle $W X Y$. Hence the point $P$ lies in the interior of the triangle $W X V$ and this is the desired triangle. If the segment $X N$ contains no point of $B$ but the segment $Y N$ contains a point of $B$, let $V$ be the first point of $B$ on the ray $X N$, and again the triangle $X W V$ is the desired triangle. If neither the segment $X N$ nor the segment $Y N$ contains a point of $B$, the point $N$ belongs to $B$ and the triangle $X W N$ is the desired triangle. The case in which the rays $Z X$ and $Y W$ have no point in common is exactly the same as the above.

Thus, in any possibility, we have established the existence of a triangle having the properties required in our theorem.

It is of interest to notice that while for any point of any bounded domain there is a triangle having the properties required in our theorem, there exist bounded domains, and in fact bounded domains with simple closed curves as boundaries, such that there is no simple polygon of more than three sides having its vertices on the boundary of the domain and so that every other point of the polygon and its interior lie in the domain. An example of such a domain is the bounded domain whose boundary is a three-cusped hypocycloid.

The University of TeXas 